\title{
REFRACTORY MACROPHAGE ACTIVATION SYNDROME AS A COMPLICATION OF RHEUMATOID ARTHRITIS AND SJÖGREN'S SYNDROME: A CASE REPORT
}

Thiago Quadrante Freitas ${ }^{1, \star}$, Maria Eugenia Teixeira Bicalho ${ }^{1}$, Janaina Baggio ${ }^{1}$, Henrique Ayres Mayrink Giardini ${ }^{1}$, Rosa Maria Rodrigues Pereira ${ }^{1}$

\author{
1.Universidade de São Paulo, São Paulo (SP), Brazil.
}

*Corresponding author: thiago.f@fm.usp.br

\section{BACKGROUND}

Macrophage activation syndrome (MAS) is a rare and potentially fatal complication of rheumatic diseases. In adults, it is associated especially with systemic lupus erythematosus and adult-onset Still's disease. Rheumatoid arthritis (RA) is responsible for $4.3 \%$ of MAS in adults, and Sjögren's syndrome (SS) for $0.9 \%$. Our objective is to describe a refractory case of MAS due to RA and SS activity.

\section{CASE REPORT}

A 63-year-old Asian Brazilian man, 60-pack year smoker, but otherwise healthy, was diagnosed with seropositive RA in 2018, and responded well to methotrexate and a short course of prednisone. Two years later, he developed recurrent fever episodes of up to $39^{\circ} \mathrm{C}$, with no specific pattern or localizing signs, and they alleviated with acetaminophen. Seven months later, after losing $7 \mathrm{~kg}$ and presenting variable periods with fever, he sought help from an infectious diseases (ID) specialist, who excluded tuberculosis, infective endocarditis, HIV, hepatitis and other prevalent IDs. An external rheumatologist added $20 \mathrm{mg}$ of prednisone to methotrexate, and later to adalimumab, due to these constitutional symptoms. However, the fever increased further, and adalimumab was discontinued. The patient was then referred to our Rheumatology service. Autoimmunity panel confirmed erosive RA and showed nuclear fine speckled 1/320 antinuclear antibody pattern, and positive anti-Ro antibody. Despite denying sicca syndrome, salivary gland biopsy lymphocyte focus score of 1.03 and positive Schirmer test confirmed SS. The PET-CT showed lung emphysema, abdominal aorta and iliac artery hypercaptation (maximum standard uptake values: 2.6 and 1.3, respectively), with diffuse atheromatosis. There were no cranial symptoms, and bilateral temporal artery ultrasonography was unremarkable. By then, the fever persisted on a weekly pattern despite prednisone and methotrexate. Fever worsened to a daily pattern, reaching $40{ }^{\circ} \mathrm{C}$, and he was admitted as an inpatient. C-reactive protein had risen to $260 \mathrm{mg} / \mathrm{L}$, with hyperferritinemia ( $7284 \mathrm{ng} / \mathrm{mL}$ ), hypertriglyceridemia, cytopenias and a new onset splenomegaly. Endocarditis, tuberculosis, leishmaniasis, malaria, cytomegalovirus, Epstein-Barr virus and parvovirus were excluded. Bone marrow evaluation confirmed MAS, and methylprednisolone pulse therapy plus cyclosporine were promptly initiated. Fever briefly receded but returned with worsening laboratory parameters. Tocilizumab was administered, fever ceased again, and laboratory parameters responded partially, but worsened two weeks later, remaining unresponsive even after treatment with immunoglobulin $(2 \mathrm{~g} / \mathrm{kg}$ ) and one etoposide cycle. The patient remains hospitalized under observation.

\section{CONCLUSION}

Rheumatoid arthritis and SS seldomly complicate with MAS. This case reminds that this complication must be considered and promptly treated. Some might be refractory to first-line treatment, with fewer therapeutic options.

\section{KEYWORDS}

Macrophage activation syndrome, Rheumatoid arthritis, Sjögren's syndrome. 